\title{
Lead-related complications after DDD pacemaker implantation
}

\author{
Maciej Dębski ${ }^{1}$, Mateusz Ulman ${ }^{1}$, Andrzej Ząbek ${ }^{1}$, Krzysztof Boczar ${ }^{1}$, Kazimierz Haberka ${ }^{1}$, \\ Marcin Kuniewicz ${ }^{1,2}$, Jacek Lelakowski ${ }^{1,3}$, Barbara Małecka ${ }^{1,3}$ \\ 1'Department of Electrocardiology, John Paul II Hospital, Krakow, Poland \\ ${ }^{2}$ Department of Anatomy, Jagiellonian University Medical College, Krakow, Poland \\ ${ }^{3}$ Institute of Cardiology, Jagiellonian University Medical College, Krakow, Poland
}

\begin{abstract}
A bstract
Background: Pacing leads remain the weakest link in pacemaker systems despite advances in manufacturing technology.

Aim: The aim of the study was to assess the long-term pacing lead performance in an unselected real-life cohort following primary DDD pacing system implantation.

Methods: A single-centre retrospective analysis of patients who underwent DDD pacing system implantation between October 1984 and December 2014 and were followed-up until August 2016 was conducted. The inclusion criterion was at least one follow-up visit after post-implant discharge. The performance of each atrial and ventricular lead implanted was evaluated during the follow-up period, and the incidence of, and predictive factors for, lead dislodgement and failure were analysed.

Results: The data of 3771 patients and 24,431.8 patient-years of follow-up were analysed. The mean follow-up of patients was $77.7 \pm 61.8$ months. During the study period, 7887 transvenous atrial and right ventricular pacing leads were implanted. Lead dislodgement occurred in 94 (1.2\%) leads (92 [2.4\%] patients), perforation in $11(0.1 \%)$ leads (10 [0.3\%] patients), and lead failure in $329(4.2 \%)$ leads (275 [7.3\%] patients). Atrial lead position was a predictive factor for lead dislodgement, while age at implantation, polyurethane $80 \mathrm{~A}$ insulation, subclavian vein access, unipolar lead construction, and lead manufacturer were multivariate predictors of lead failure.

Conclusions: Leads with polyurethane $80 \mathrm{~A}$ insulation, unipolar construction, and those implanted via subclavian vein puncture exhibited the worst long-term performance.
\end{abstract}

Key words: risk factors, cardiac pacing, pacing lead, complications, lead failure

Kardiol Pol 2018; 76, 8: 1224-1231

\section{INTRODUCTION}

Permanent pacemaker technology has evolved considerably over the past five decades. Major advancements have been made in the design of pacing leads for improved electrical performance and patient safety. However, despite significant progress, pacing leads have remained the most vulnerable component of transvenous pacing systems. Lead-related complications are the primary reason for reoperation following cardiac-implantable electronic device implantations [1, 2]. Management of displaced and failed leads may require challenging lead extraction procedures with surgical backup and transoesophageal echocardiography monitoring [3, 4]. Further- more, lead-related interventions are associated with increased patient morbidity and healthcare costs [5]. The short-term performance of pacing leads and predictors for early lead-related complications have been evaluated in many reports [1, 5-7]. However, unlike implantable cardioverter-defibrillator (ICD) leads [8, 9], the long-term reliability and chronic performance of DDD pacing leads have received less attention. Thus, the objectives of the present analysis were to examine whether the progress in lead construction technology has affected the rate of lead-related complications in an unselected real-life cohort implanted with a DDD pacemaker, and to identify the predictors of lead dislodgement and lead failure in a long-term follow-up. 


\section{METHODS}

The study cohort comprised all consecutive patients who underwent primary DDD pacemaker implantation at a high-volume tertiary reference university implantation centre between 4 October 1984 (the first performed DDD pacemaker implantation at the Department of Electrocardiology, John Paul II Hospital, Kraków, Poland) and 31 December 2014. The study inclusion criterion was at least one follow-up visit after hospital discharge. Patients who were permanently lost to follow-up after hospital discharge were excluded from the study. The follow-up was completed on 31 August 2016. The data used in the analysis included the following: (1) patients' demographic baseline characteristics (date of birth, age at primary DDD implantation, age at additional lead implantation procedure, and sex); (2) the type of venous access (subclavian vein puncture or cephalic vein cut-down); (3) the name of the endocardial pacemaker lead model; (4) the lead manufacturer; (5) the position of the lead (atrial or ventricular); (6) the type of fixation (passive or active); (7) lead polarity (unipolar or bipolar); (8) the type of lead dysfunction (lead dislodgement, cardiac perforation, or lead failure); (9) the date of the diagnosis of lead-related complications; (10) the length of lead follow-up, and (11) the length of patient follow-up (i.e. time from primary DDD pacemaker implantation to the last follow-up visit).

Retrospective data generated on the basis of this analysis were retrieved from paper and electronic medical records issued during all hospitalisations in our department, other departments within our hospital, and from outpatient clinic medical files comprising pacemaker follow-up visits as well as the documentation of device-related procedures performed in other cardiology centres. Lead dislodgement was defined as a need for operative adjustment, replacement, or surgical abandonment of the lead because of macroscopic dislodgement. Cardiac perforation was defined as radiographic evidence of the excursion of the pacing lead into the pericardial sac, with abnormal echocardiography and/or cardiac computed tomography findings indicative of a perforation. Lead failure due to lead damage was defined as an inappropriately elevated pacing threshold or detection of problems that persisted despite device reprogramming and/or out-of-limit alterations in lead impedance $(<200 \Omega$ or $>2000 \Omega)$. Lead failure was considered only when lead dislodgement and cardiac perforation were ruled out during diagnostic work-up. Reoperation was not a prerequisite for diagnosing lead failure. During most of the study period, implantations were performed by a small number of highly experienced cardiologists. Unipolar leads were implanted between 1984 and 2002, and bipolar leads were implanted since 1988. Cephalic cut-down technique was used for venous access whenever possible. The subclavian vein was directly punctured when the preparation of the cephalic vein failed or the insertion of the second lead was impossible despite the application of a modified cephalic vein guidewire technique [10].
Axillary vein puncture has not been used in our centre. All transvenous atrial and ventricular pacing leads implanted were examined during the follow-up period. The performance of right ventricular cardioverter-defibrillator leads, right ventricular VDD leads, transvenous left ventricular leads, and epicardial leads implanted in our patient cohort was not assessed in the present study. The duration of lead follow-up was defined as the period between lead implantation and whichever of the following that occurred first: total lead fracture visible on chest X-rays with ineffectual capture/no sensing and abnormal impedance at device interrogation, surgical lead abandonment, lead extraction, or the last follow-up visit.

\section{Statistical analysis}

The data were evaluated using IBM SPSS Statistics for Windows, version 25 (IBM Corp., Armonk, NY, USA). Normality was tested using Shapiro-Wilk test (S-W) for samples $\leq 2000$ and Kolmogorov-Smirnov test (K-S) for samples $>$ 2000. Continuous variables are expressed as mean \pm standard deviation and additionally as median and interquartile range (IQR) for variables with non-normal distribution. Categorical variables were compared using $\chi^{2}$ test. Cumulative survival rates were calculated according to the Kaplan-Meier method. Event times were measured from the time of each lead implantation procedure. The significance of differences between the considered variables in terms of lead survival was assessed using the log-rank test. The prognostic impact of selected variables on lead dislodgement was assessed using univariate logistic regression model. The association between selected variables and lead failure was assessed using the Cox proportional hazards model. A p-value of $<0.05$ was considered significant.

\section{RESULTS}

During the study period, 3932 consecutive patients underwent primary DDD pacemaker implantation. After hospital discharge, 161 patients were lost to follow-up and were thereby excluded from the analysis. The data of 3771 patients and $24,431.8$ patient-years of follow-up were analysed. The mean duration of follow-up was $77.7 \pm 61.8$, median 62.5 , IQR 78.2 months (max. 369.5 months) (K-S; $\mathrm{p}<0.001$ ). During the study period, 7887 transvenous atrial and right ventricular pacing leads were implanted: 7542 during initial DDD pacemaker implantation and 345 during device revisions after either lead explantation or lead abandonment; 3060 (38.8\%) leads had passive fixation and 4827 (61.2\%) leads had active fixation; 349 (4.4\%) leads were unipolar and 7538 (95.6\%) leads were bipolar. Atrial lead fixation was passive in 1533 (38.7\%) leads and active in 2428 (61.7\%) leads, whereas ventricular lead fixation was passive in 1527 (38.9\%) leads and active in 2399 (61.8\%) leads. The lead model names, manufacturers, types of insulation and the exact number of leads and lead failures are summarised in Table 1 . The mean patient age at lead implantation was $69.8 \pm 12.1$ (15.2-96.6), median 
Table 1. Lead manufacturers, lead model names with their insulation, polarity and position, number, and rate of lead failure. If not specified, the lead insulation was silicone

\begin{tabular}{|c|c|c|c|c|c|c|c|c|}
\hline Manufacturer & $\begin{array}{l}\text { Lead } \\
\text { model }\end{array}$ & Insulation & Unipolar & Bipolar & Atrial & Ventricular & $\begin{array}{l}\text { Lead } \\
\text { failure }\end{array}$ & $\begin{array}{c}\text { Failure rate } \\
\text { [\%] }\end{array}$ \\
\hline \multirow[t]{35}{*}{ Biotronik } & DJP & & + & & 34 & & 9 & 26.5 \\
\hline & DN/DNR & & + & & & 20 & 7 & 35 \\
\hline & Elox & & & + & 7 & & 1 & 14.3 \\
\hline & & & & + & & 15 & 3 & 20 \\
\hline & $J P$ & & & + & 24 & & 1 & 4.2 \\
\hline & PE 60/4-DN & & + & & & 13 & 8 & 61.5 \\
\hline & PE 603-DJ & & + & & 14 & & 9 & 64.3 \\
\hline & Polyrox J & & & + & 50 & & 3 & 6 \\
\hline & Polyrox & & & + & & 29 & 0 & 0 \\
\hline & Retrox J & & & + & 4 & & 0 & 0 \\
\hline & SD & & & + & & 12 & 2 & 16.7 \\
\hline & Selox SR & & & + & 25 & & 0 & 0 \\
\hline & & & & + & & 22 & 1 & 4.5 \\
\hline & Selox ST & & & + & & 91 & 3 & 3.3 \\
\hline & Selox JT & & & + & 98 & & 1 & 1 \\
\hline & Setrox S & & & + & 152 & & 2 & 1.3 \\
\hline & & & & + & & 138 & 2 & 1.4 \\
\hline & Siello & & & + & 327 & & 0 & 0 \\
\hline & & & & + & & 296 & 1 & 0.3 \\
\hline & Solia & & & + & 17 & & 0 & 0 \\
\hline & & & & + & & 17 & 0 & 0 \\
\hline & SR-60-UP & & + & & 1 & & 0 & 0 \\
\hline & & & + & & & 1 & 0 & 0 \\
\hline & Synox & & & + & 688 & & 19 & 2.8 \\
\hline & & & & + & & 644 & 14 & 2.2 \\
\hline & TIJ & & + & & 102 & & 21 & 20.6 \\
\hline & & & & + & 379 & & 31 & 8.2 \\
\hline & TIR & & + & & & 123 & 10 & 8.1 \\
\hline & & & & + & & 412 & 20 & 4.9 \\
\hline & Y & & & + & 9 & & 1 & 11.1 \\
\hline & & & & + & & 4 & 0 & 0 \\
\hline & YP & & + & & 1 & & 0 & 0 \\
\hline & & & + & & & 1 & 0 & 0 \\
\hline & & & & + & 1 & & 0 & 0 \\
\hline & & & & + & & 10 & 0 & 0 \\
\hline Biotronik failure rate [\%] & & & 20.6 & 3 & 5.1 & 3.8 & & 4.5 \\
\hline \multirow[t]{10}{*}{ Medtronic } & 4082 & PU 80A & & + & & 1 & 0 & 0 \\
\hline & 6957 & PU 80A & + & & 2 & & 1 & 50 \\
\hline & 4004/4004M & PU 80A & & + & & 14 & 8 & 57.1 \\
\hline & $4504 \mathrm{M}$ & PU 80A & & + & 15 & & 2 & 13.3 \\
\hline & 4068 & PU 80A & & + & & 1 & 0 & 0 \\
\hline & 4557 & PU 80A & + & & 5 & & 0 & 0 \\
\hline & $5024 \mathrm{M}$ & & & + & & 6 & 0 & 0 \\
\hline & $5524 \mathrm{M}$ & & & + & 7 & & 1 & 14.3 \\
\hline & 5054 & & & + & & 8 & 0 & 0 \\
\hline & 5554 & & & + & 12 & & 1 & 8.3 \\
\hline
\end{tabular}


Table 1. (cont.) Lead manufacturers, lead model names with their insulation, polarity and position, number, and rate of lead failure. If not specified, the lead insulation was silicone

\begin{tabular}{|c|c|c|c|c|c|c|c|c|}
\hline Manufacturer & $\begin{array}{l}\text { Lead } \\
\text { model }\end{array}$ & Insulation & Unipolar & Bipolar & Atrial & Ventricular & $\begin{array}{l}\text { Lead } \\
\text { failure }\end{array}$ & $\begin{array}{c}\text { Failure rate } \\
\text { [\%] }\end{array}$ \\
\hline \multirow[t]{8}{*}{ Medtronic } & 5068 & & & + & & 1 & 0 & 0 \\
\hline & 5568 & & & + & 51 & & 0 & 0 \\
\hline & 4076 & PU 55D & & + & 6 & & 0 & 0 \\
\hline & & PU 55D & & + & & 8 & 0 & 0 \\
\hline & 5076 & & & + & 1519 & & 51 & 3.4 \\
\hline & & & & + & & 1398 & 26 & 1.9 \\
\hline & 5092 & & & + & & 129 & 4 & 3.1 \\
\hline & 5592 & & & + & 93 & & 4 & 4.3 \\
\hline Medtronic failure rate [\%] & & & 14.3 & 3 & 3.5 & 2.4 & & 3 \\
\hline \multirow[t]{2}{*}{ Vitatron } & ICF09B & & & + & 292 & & 31 & 10.6 \\
\hline & & & & + & & 484 & 19 & 3.9 \\
\hline Vitatron failure rate [\%] & & & & 6.4 & 10.6 & 3.9 & & 6.4 \\
\hline \multirow[t]{4}{*}{ Other } & & & + & & 11 & & 5 & 45.5 \\
\hline & & & + & & & 21 & 6 & 28.6 \\
\hline & & & & + & 15 & & 1 & 0.7 \\
\hline & & & & + & & 7 & 0 & 0 \\
\hline Total & & & 349 & 7538 & 3961 & 3926 & 329 & 4.2 \\
\hline
\end{tabular}

PU - polyurethane

71.9, IQR 14.2 years (K-S; $\mathrm{p}<0.001$ ). Considering each lead placement separately, 4210 (53.4\%) leads were implanted in male patients and 3677 (46.6\%) in female patients. The mean duration of lead follow-up was $73.2 \pm 59.5$, median 58.3, IQR 73.1 months (max. 369.5 months) (K-S; $\mathrm{p}<0.001$ ).

Subclavian vein access was used for 4950 (62.8\%) leads and cephalic vein access for 2937 (37.2\%) leads. Ventricular leads were more frequently inserted via the cephalic vein (2379 [60.6\%]) than via the subclavian vein (1546 [39.4\%]), whereas atrial leads were more frequently inserted via the subclavian vein (3404 [85.9\%]) than via the cephalic vein (558 [14.1\%]). The choice of venous access was not associated with patient's $\operatorname{sex}\left(\chi^{2}=1.35 ; p=0.245\right)$.

Lead dysfunction occurred in 434 (5.5\%) leads: 11 (0.1\%) were confirmed to have perforated the heart, 94 (1.2\%) dislodged, and 329 (4.2\%) failed. Lead dislodgement occurred in $92(2.4 \%)$ patients, perforation in $10(0.3 \%)$ patients, and lead failure in 275 (7.3\%) patients. Regarding manufacturers, $50(1.3 \%)$ of the dislodged leads were manufactured by Biotronik (Berlin, Germany), 34 (1\%) by Medtronic (Minneapolis, MN, USA), and 10 (1.3\%) by Vitatron (Maastricht, Netherlands); furthermore, 169 (4.5\%) of the failed leads were manufactured by Biotronik, 98 (3\%) by Medtronic, 50 (6.4\%) by Vitatron, and $12(22.2 \%)$ by other manufacturers.

Predictors of lead dislodgement and lead failure are shown in Figures 1 and 2, respectively.

\section{Predictors of lead dislodgement}

In the univariate logistic regression analysis performed at lead implantation, atrial lead position was the only independent predictor of lead dislodgement. The association between lead dislocation and patient's sex, age at lead implantation (one-year increase), and the type of lead fixation showed no deviation from linearity.

\section{Predictors of lead failure}

Independent predictors of lead failure measured at lead implantation in the multivariable Cox regression model were subclavian vein puncture, unipolar lead construction, age at the time of implantation, and lead manufacturer. The age at the time of implantation inversely influenced lead failure, with younger patients having a significantly higher likelihood of lead failure (odds ratio 0.985; $p<0.001$ ). With Medtronic leads considered as a reference, Vitatron leads were significantly associated with an increased risk of lead failure, whereas Biotronik leads significantly protected against lead failure. Neither lead position (atrial or ventricular) nor patient's sex influenced lead failure.

Actuarial failure-free lead survival rates of unipolar leads five, 10, 15, and 20 years after lead implantation were $90 \%, 81 \%, 74 \%$, and $70 \%$, respectively, and those of bipolar leads were 98\%, 94\%, 90\%, and 86\%, respectively (log-rank $\chi^{2}=57.253 ; p<0.001 ;$ Fig. 3 ). To avoid bias related to the 


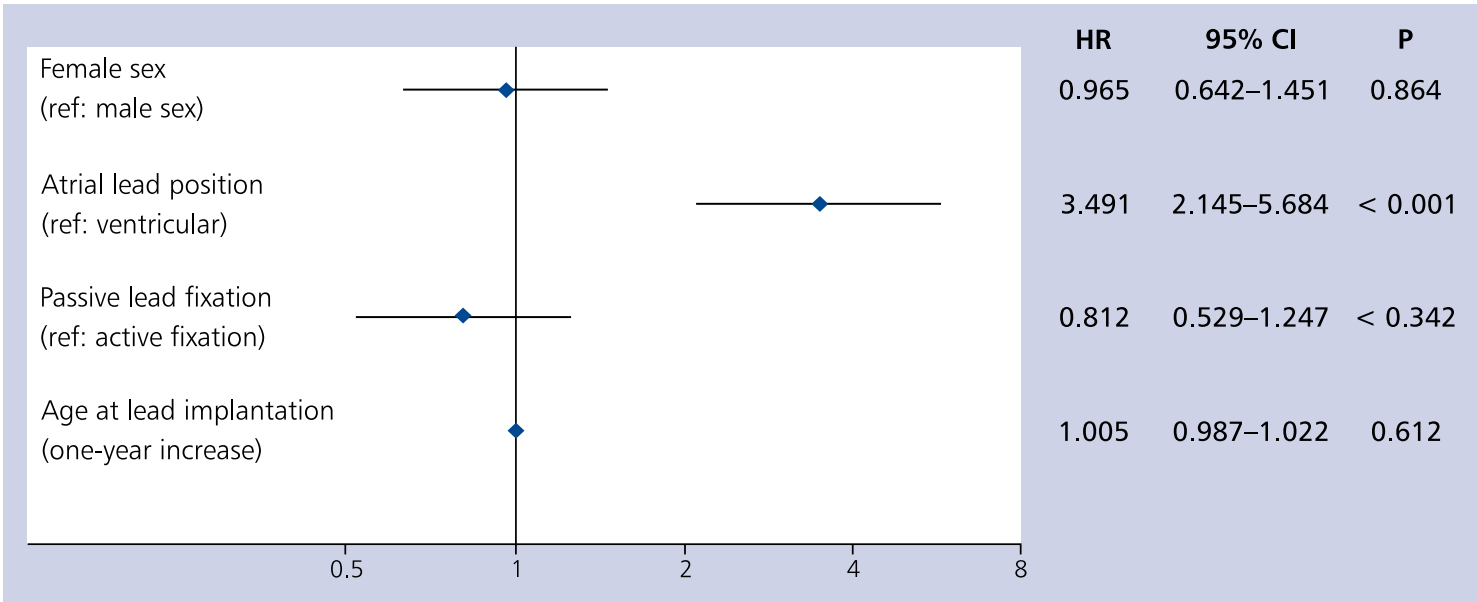

Figure 1. Univariate logistic regression analysis of lead dislodgement; $\mathrm{Cl}$ — confidence interval; $\mathrm{HR}$ — hazard ratio

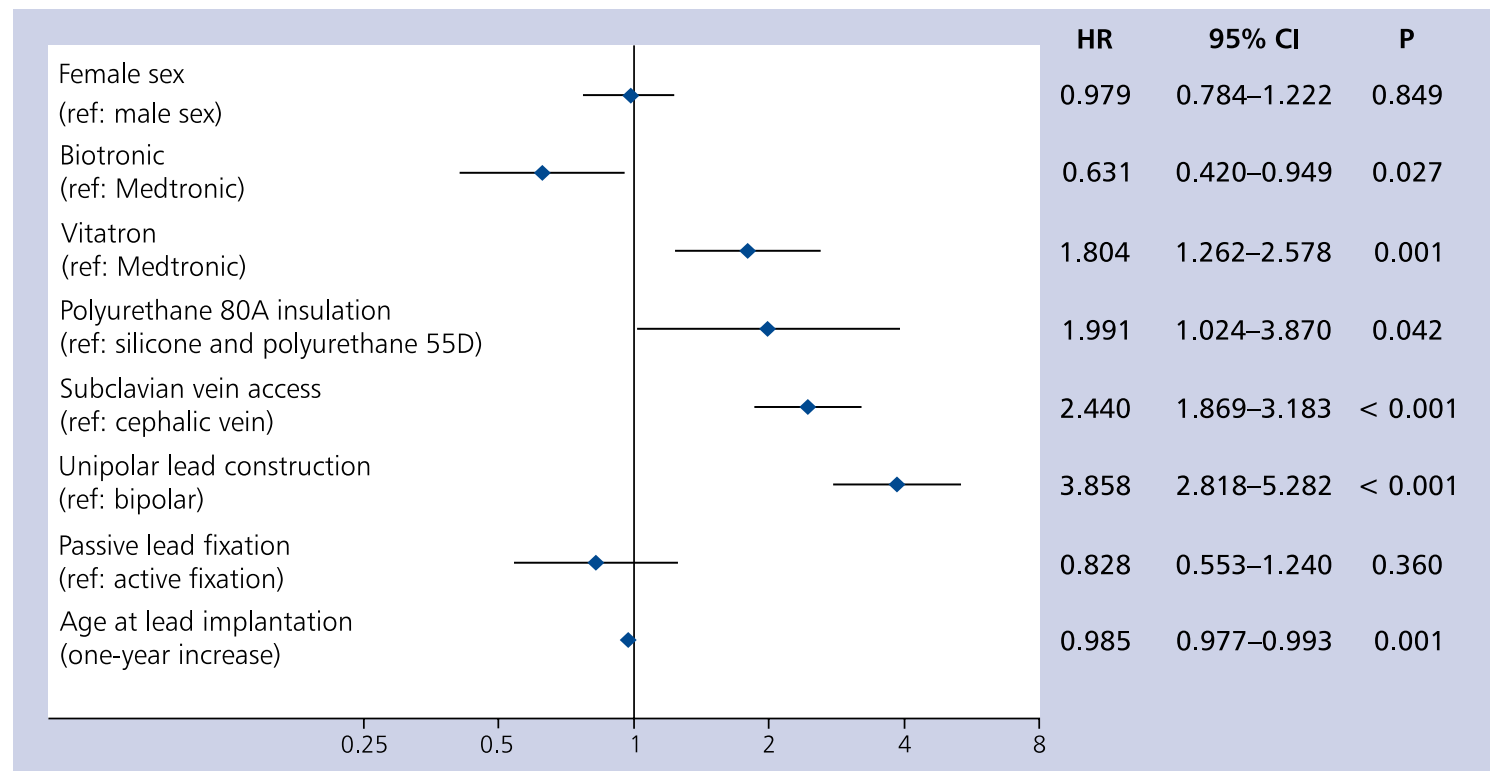

Figure 2. Multivariable Cox regression analysis of lead failure in all Biotronik, Medtronic, and Vitatron leads (7833 leads); $\mathrm{Cl}$ - confidence interval; $\mathrm{HR}$ - hazard ratio

comparison of old unipolar leads with some modern bipolar leads, a subanalysis of leads implanted only between 1988 and 2002 (i.e. between the year of first bipolar lead implantation and the year of last unipolar lead implantation) at our centre was performed. The analysis confirmed that the performance of 337 unipolar leads remained significantly inferior to that of 1596 bipolar leads (log-rank $\chi^{2}=61.987$; $p<0.001$ ). In the entire cohort, lead failure occurred at a mean duration of $73.6 \pm$ 53.3, median 61.0, IQR 64.4 months after implantation (S-W; $\mathrm{p}<0.001)$. Biotronik bipolar leads failed after $81.6 \pm 57$, median 81.4, IQR 83.4 months $(S-W ; p<0.003)$ whereas Biotronik unipolar leads failed after $90.4 \pm 71.6$, me- dian 63.3, IQR 99.1 months (S-W; p < 0.001); Medtronic and Vitatron bipolar leads failed after $61.6 \pm 40.4$, median 52.3, IQR 49.3 months (S-W; $p<0.001)$ and $57.1 \pm 29.2$ months $(\mathrm{S}-\mathrm{W} ; \mathrm{p}=0.439)$, respectively. Leads insulated with polyurethane $80 \mathrm{~A}$ showed an almost fourfold higher incidence of failure than those insulated with silicone (26.5 vs. 6.8 per 1000 lead-years of follow-up). Among leads insulated with polyurethane 55D, we did not observe lead failure during a mean of $49.1 \pm 18.5$, median 57.3, IQR 23.7 months of follow-up (S-W; $\mathrm{p}<0.012)$. When considering only bipolar leads with silicone insulation (Fig. 4), the performance of Biotronik leads was significantly superior to that of Medtronic 


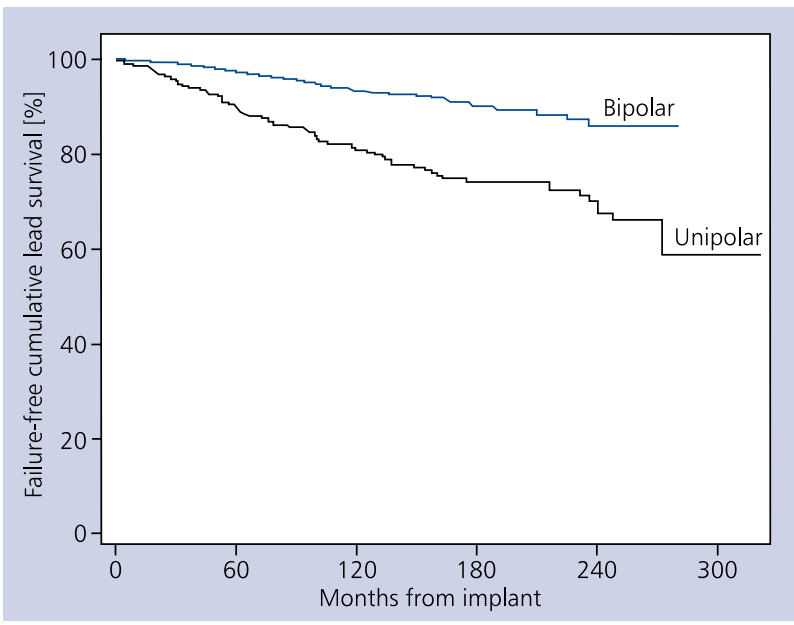

Figure 3. Cumulative lead performance by polarity. Differences between groups are significant: log-rank $\chi^{2}=75.267 ; p<0.001$

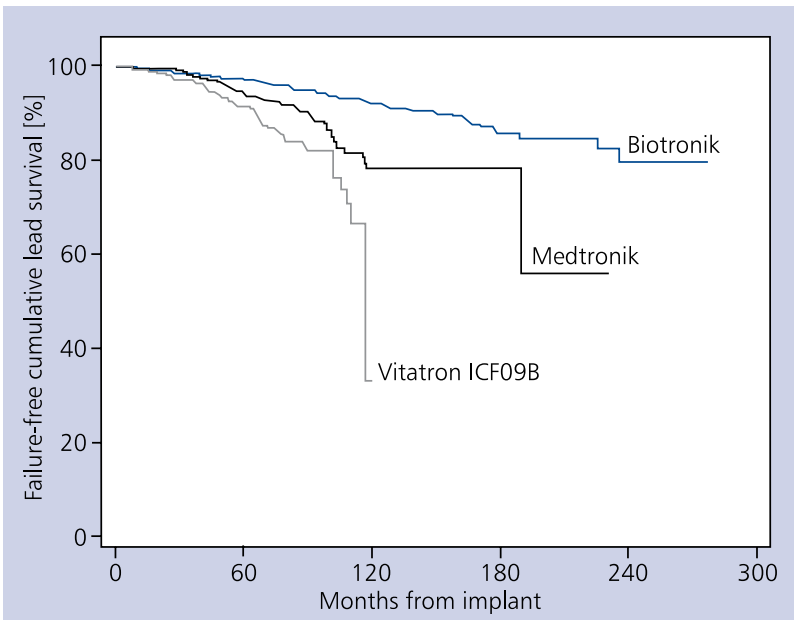

Figure 4. Kaplan-Meier failure-free survival estimate of bipolar leads with silicone insulation according to manufacturer. Number of leads: Biotronik - 3471, Medtronic - 3224, Vitatron - 776. Differences between all groups are significant: Biotronik vs. Medtronic (log-rank $\left.\chi^{2}=21.041 ; p<0.001\right)$; Medtronic vs. Vitatron (log-rank $\left.\chi^{2}=9.603 ; p=0.002\right)$; Biotronik vs. Vitatron (log-rank $\chi^{2}=55.035 ; p<0.001$ )

leads ( $p<0.001$ ), and the performance of Medtronic leads was significantly better than that of Vitatron ICF09B leads $(p=0.002)$. The incidence of failure in bipolar silicone-insulated leads was 4.1 per 1000 lead-years of follow-up for Biotronik leads, 6.5 per 1000 lead-years of follow-up for Medtronic leads, and 12.2 per 1000 lead-years of follow-up for Vitatron leads. The largest group of modern bipolar active-fixation leads in our study was the Medtronic model 5076 (2917 leads), with a six-year survival rate of $95.6 \%$.

\section{DISCUSSION}

One of the main findings of this study is the very long-term data regarding the performance of several types of endocardial pacing leads. In the present study, the overall performance of the bipolar leads was significantly better than that of the unipolar leads. Ten-year survival rates of $94 \%$ for bipolar leads and $81 \%$ for unipolar leads were reported. The significant difference persisted when the bipolar leads were compared with the unipolar leads implanted in the same period. Our data concur with a previously published report by Helguera et al. [11], who assessed 2611 leads implanted between 1980 and 1991 and observed a tendency towards a better 10-year survival among unipolar leads compared with bipolar leads. Furthermore, a register-based report on $>33,000$ endocardial pacing leads implanted in Denmark between 1982 and 1999 revealed that the 10-year survival rate of unipolar leads was significantly better than that of bipolar leads despite the exclusion of several poorly performing bipolar leads from the analysis [12]. However, the authors noted that the performance of bipolar leads had been improving and that some models achieved an overall survival rate of $>95 \%$ at six years. The largest group of modern bipolar leads in our study was the Medtronic model 5076, with a six-year failure-free lead survival rate of $95.6 \%$. Interestingly, this value is lower than the officially reported lead survival rate of approximately $98.5 \%$, according to the Manufacturer's Product Surveillance Registry [13]. Of note, the rate demonstrated by Medtronic encompasses all the complications, including cardiac perforations and lead dislodgements, which were excluded from the present lead failure analysis [13]. According to the Swedish Pacemaker Registry, the 10-year survival rate of pacemaker leads was 98\% [14]. The median time to failure for all leads in our study was 6.1 years. The analysis of the causes of lead failure in 617 explanted failed leads indicated a mean time to failure of $7.2 \pm 5.2$ years [15]. Notably, both Hauser et al. [15] and Arnsbo et al. [12] qualified a lead as failed only when surgical intervention was performed, whereas we did not require reoperation as a clinical criterion to diagnose lead failure.

The data regarding the better survival of Biotronik leads compared with Medtronic leads and the worst survival of Vitatron ICF09B leads should be interpreted with caution and verified in further prospective studies. The poor performance of the early Medtronic leads 4004/M and 4504 has been associated with the use of polyurethane P80A as the insulation material. Polyurethane leads have been implanted in humans since 1977 [16]. Within four years from the first use of polyurethane $80 \mathrm{~A}$ in pacing leads, the insulation material was found to exhibit surface cracks and clinical evidence of insulation failure. The high rate of polyurethane $80 \mathrm{~A}$ insulation failure was observed in coaxial bipolar leads but not in unipolar leads [17]. Defects have also been confirmed in Medtronic leads 4082,6972 , and 4012 . This has led to the use of another 
polymer, polyurethane 55D, which is a more reliable material [18]. Insulation with polyurethane $80 \mathrm{~A}$ was an independent predictor of lead failure. This observation is in agreement with other reports on long-term lead performance [12, 15, 19].

Another variable independently associated with lead failure was subclavian vein puncture. Subclavian crush syndrome is a well-described cause of pacemaker lead failure resulting from entrapment of a lead or leads between the clavicle and the first rib. Infraclavicular lead failure has been associated with soft tissue entrapment in the subclavius muscle or the costoclavicular ligament, where the lead undergoes repeated episodes of bending and tension during pectoral muscle movements [20]. However, whether one technique is superior to the other remains debatable [11, 21, 22]. On the basis of the records of 409 patients implanted with $681 \mathrm{im}$ plantable pacemaker leads and $20(2.9 \%)$ lead failures at a mean of $73.6 \pm 33.1$ months of follow-up, Chan et al. [22] recommended axillary vein puncture instead of cephalic vein cut-down as the venous access of choice for pacemaker lead implantation. Conversely, in the analysis of Sprint Fidelis lead failures, Birnie et al. [9] demonstrated that both axillary and subclavian accesses increased the hazard of failure compared with cephalic access. Reportedly, the need for subclavian vein puncture independently increased the mean implantation and fluoroscopy times [23]. Our data are strongly supportive of the use of cephalic vein cut-down as the first choice of venous access and advocate against using subclavian vein puncture when possible. Importantly, implanting three leads via the cephalic vein for a cardiac resynchronisation therapy pacemaker or defibrillator has been demonstrated to be a safe and effective first approach technique [24].

Regarding the fixation mechanism, in a study investigating the predictors of lead fracture in children, Olgun et al. [25] detected an association between the active fixation mechanism and lead fractures and postulated that the repeated screwing-retraction attempts during implantation may have a deleterious effect on the inner coil and consequently weaken the lead body. In contrast, Hauser et al. [15] reported that active and passive-fixation mechanisms were equally represented among leads with malfunction. In the present study, the lead fixation mechanism did not predict lead failure or dislodgement. This is in agreement with a benchmark study reporting short-term complications arising after DDD pacemaker implantation, in which there was no association between the fixation type of atrial or ventricular leads and the occurrence of atrial and ventricular lead dislodgement or any lead-related complication [6, 26]. Additionally, no association was noted between female sex and increased risk of failures. A strong correlation between female sex and increased risk of failure has been demonstrated for various ICD leads [8, 9]. Finally, with each one-year increase in patient age, the risk of lead failure decreased. This observation is in accordance with the results of the FOLLOWPACE study [26] and may be attributed to the increased physical activity of younger patients [27] as well as to the bias related to longer life expectancy and follow-up duration in younger patients.

The main limitation is the retrospective and single-centre design of this study, with all the inherent shortcomings. First, because the follow-up visits predominantly occurred once a year, a certain number of lead-related complications after the last pacemaker interrogation might not have been detected. To address this limitation, large prospective studies with telemonitoring are warranted. Second, the fact that we analysed the performance of 39 lead models used in different years over a three-decade study period rendered the lead model follow-up heterogeneous, and the number of patients remains too small to demonstrate clinically important differences in complication rates between various lead types. Finally, constant innovations in this field lead to a dynamism wherein it is difficult to apply conclusions regarding technologies that are no longer used.

In conclusion, we assessed the long-term performance of 7887 transvenous atrial and right ventricular leads in a 10-year follow-up and determined survival rates of $81 \%$ for unipolar leads and $94 \%$ for bipolar leads. During 24,431.8 patient-years of follow-up, lead failure occurred in 275 (7.3\%) patients and lead dislodgement in 92 (2.4\%) patients. Atrial lead position predicted lead dislodgement, whereas fixation type, patient sex, or age at the time of implantation did not predict dislocation. Lead manufacturer, unipolar construction, subclavian vein access, insulation with polyurethane $80 \mathrm{~A}$, and young age of patients were independently associated with lead failure.

Conflict of interest: Maciej Dębski received travel expenses coverage from Biotronik and Medtronic; Mateusz Ulman — no conflict of interest; Andrzej Ząbek received travel expenses coverage from Biotronik, Medtronic, and St. Jude Medical; Krzysztof Boczar received travel expenses coverage from Biotronik, Medtronic, and St. Jude Medical; Kazimierz Haberka - no conflict of interest; Marcin Kuniewicz received travel expenses coverage from Medtronic; Jacek Lelakowski - no conflict of interest; Barbara Małecka received travel expenses coverage from Biotronik, Boston Scientific, Medtronic, and St. Jude Medical.

\section{References}

1. Kirkfeldt RE, Johansen JB, Nohr EA, et al. Risk factors for lead complications in cardiac pacing: a population-based cohort study of 28,860 Danish patients. Heart Rhythm. 2011; 8(10): 1622-1628, doi: 10.1016/j.hrthm.2011.04.014, indexed in Pubmed: 21699827

2. Zabek A, Malecka B, Haberka K, et al. The analysis of indications and early results of transvenous lead extraction in patients with a pacemaker, ICD and CRT - single-center experience. Acta Cardiol. 2015; 70(6): 685-692, doi: 10.2143/AC.70.6.3120181, indexed in Pubmed: 26717217.

3. Boczar K, Komar M, Ząbek A, et al. Spontaneous dislocation of the endocardial lead into the left ventricle through the intraventricular septum. Kardiol Pol. 2017; 75(1): 79, doi: 10.5603/KP.2017.0004, indexed in Pubmed: 28124788. 
4. Ząbek A, Boczar K, Dębski M, et al. Successful transvenous extraction of an endocardial lead more than 35 years old using mechanical systems. Kardiol Pol. 2016; 74(11): 1357, doi: 10.5603/KP.2016.0161, indexed in Pubmed: 27910081.

5. Kirkfeldt RE, Johansen JB, Nohr EA, et al. Complications after cardiac implantable electronic device implantations: an analysis of a complete, nationwide cohort in Denmark. Eur Heart J. 2014; 35(18): 1186-1194, doi: 10.1093/eurheartj/eht511, indexed in Pubmed: 24347317.

6. Ellenbogen KA, Hellkamp AS, Wilkoff BL, et al. Complications arising after implantation of DDD pacemakers: the MOST experience. Am J Cardiol. 2003; 92(6): 740-741, indexed in Pubmed: 12972124

7. Pakarinen S, Oikarinen L, Toivonen L. Short-term implantation-related complications of cardiac rhythm management device therapy: a retrospective single-centre 1-year survey. Europace. 2010; 12(1): 103-108, doi: 10.1093/europace/eup361, indexed in Pubmed: 19914920.

8. Kleemann T, Becker T, Doenges K, et al. Annual rate of transvenous defibrillation lead defects in implantable cardioverter-defibrillators over a period of $>10$ years. Circulation. $2007 ; 115(19)$ : 2474-2480, doi: 10.1161/CIRCULATIONAHA.106.663807, indexed in Pubmed: 17470696.

9. Birnie DH, Parkash R, Exner DV, et al. Clinical predictors of Fidelis lead failure: report from the Canadian Heart Rhythm Society Device Committee. Circulation. 2012; 125(10): 1217-1225, doi: 10.1161/CIRCULATIONAHA.111.053744, indexed in Pubmed: 22311781

10. Ong LS, Barold SS, Lederman M, et al. Cephalic vein guide wire technique for implantation of permanent pacemakers. Am Heart J. 1987; 114(4 Pt 1): 753-756, indexed in Pubmed: 3661365.

11. Helguera M, Maloney J, Pinski S, et al. Long-term performance of endocardial pacing leads. Pacing Clin Electrophysiol. 1994; 17(1): 56-64, doi: 10.1111/j.1540-8159.1994.tb01351.x.

12. Arnsbo P, Møller M. Updated appraisal of pacing lead performance from the Danish Pacemaker Register: the reliability of bipolar pacing leads has improved. Pacing Clin Electrophysiol. 2000; 23(9): 1401-1406, indexed in Pubmed: 11025898.

13. http://wwwp.medtronic.com/productperformance/ model/5076-capsurefix-novus.html (accessed in August 2017).

14. Gadler F, Valzania C, Linde C. Current use of implantable electrical devices in Sweden: data from the Swedish pacemaker and implantable cardioverter-defibrillator registry. Europace. 2015; 17(1): 69-77, doi: 10.1093/europace/euu233, indexed in Pubmed: 25336667.

15. Hauser RG, Hayes DL, Kallinen LM, et al. Clinical experience with pacemaker pulse generators and transvenous leads: an 8-year prospective multicenter study. Heart Rhythm. 2007; 4(2): 154-160, doi: 10.1016/j.hrthm.2006.10.009, indexed in Pubmed: 17275749 .
16. Stokes KB, Church T. Ten-year experience with implanted polyurethane lead insulation. Pacing Clin Electrophysiol. 1986; 9(6 Pt 2): 1160-1165, indexed in Pubmed: 2432526.

17. Rosenheck S, Sharon Z, Leibowitz D. Artifacts recorded through failing bipolar polyurethane insulated permanent pacing leads. Europace. 2000; 2(1): 60-65, doi: 10.1053/eupc.1999.0062, indexed in Pubmed: 11225597.

18. Ellenbogen KA, Wood MA. Cardiac Pacing and ICDs. 2011.

19. Hayes D, Graham K, Irwin M, et al. Multicenter experience with a bipolar tined polyurethane ventricular lead. Pacing Clin Electrophysiol. 1995; 18(5): 999-1004, doi: 10.1111/j.15408159.1995.tb04740.x.

20. Magney JE, Flynn DM, Parsons JA, et al. Anatomical mechanisms explaining damage to pacemaker leads, defibrillator leads, and failure of central venous catheters adjacent to the sternoclavicular joint. Pacing Clin Electrophysiol. 1993; 16(3 Pt 1): 445-457, indexed in Pubmed: 7681196.

21. Calkins H, Ramza BM, Brinker J, et al. Prospective randomized comparison of the safety and effectiveness of placement of endocardial pacemaker and defibrillator leads using the extrathoracic subclavian vein guided by contrast venography versus the cephalic approach. Pacing Clin Electrophysiol. 2001; 24(4 Pt 1): 456-464, indexed in Pubmed: 11341082.

22. Chan NY, Kwong NP, Cheong AP. Venous access and long-term pacemaker lead failure: comparing contrast-guided axillary vein puncture with subclavian puncture and cephalic cutdown. Europace. 2017; 19(7): 1193-1197, doi: 10.1093/europace/euw147, indexed in Pubmed: 27733455.

23. Eberhardt F, Bode F, Bonnemeier H, et al. Long term complications in single and dual chamber pacing are influenced by surgical experience and patient morbidity. Heart. 2005; 91(4): 500-506, doi: 10.1136/hrt.2003.025411, indexed in Pubmed: 15772212.

24. Hadjis A, Proietti R, Essebag V. Implantation of cardiac resynchronization therapy devices using three leads by cephalic vein dissection approach. Europace. 2017; 19(9): 1514-1520, doi: 10.1093/europace/euw276, indexed in Pubmed: 28340223.

25. Olgun H, Karagoz T, Celiker A, et al. Patient- and lead-related factors affecting lead fracture in children with transvenous permanent pacemaker. Europace. 2008; 10(7): 844-847, doi: 10.1093/europace/eun109, indexed in Pubmed: 18448424.

26. Udo EO, Zuithoff NPA, van Hemel NM, et al. Incidence and predictors of short- and long-term complications in pacemaker therapy: the FOLLOWPACE study. Heart Rhythm. 2012; 9(5): 728-735, doi: 10.1016/j.hrthm.2011.12.014, indexed in Pubmed: 22182495

27. Morrison TB, Rea RF, Hodge DO, et al. Risk factors for implantable defibrillator lead fracture in a recalled and a nonrecalled lead. J Cardiovasc Electrophysiol. 2010; 21(6): 671-677, doi: 10.1111/j.1540-8167.2009.01683.x, indexed in Pubmed: 20082653.

Cite this article as: Dębski M, Ulman M, Ząbek A, et al. Lead-related complications after DDD pacemaker implantation. Kardiol Pol. 2018; 76(8): 1224-1231, doi: 10.5603/KP.a2018.0089.

\section{WHAT IS NEW?}

A retrospective analysis was conducted in consecutive patients who were implanted with dual-chamber pacemakers at a tertiary reference cardiology centre between 1984 and 2014 and were followed up in an outpatient pacemaker clinic until 2016. With the data of 3771 patients and 24,431.8 patient-years of follow-up, this is one of the largest single-centre analyses of lead-related complications. Lead dislodgement occurred in $1.2 \%$ of leads, and lead failure occurred in $4.2 \%$ of leads. Atrial lead position was associated with dislodgement, whereas the type of lead fixation, age, and sex did not affect the rate of dislodgement. Independent predictors of lead failure included subclavian vein puncture, unipolar lead construction, age at the time of implantation, and lead manufacturer. Moreover, this study compared the outcomes of Medtronic and Biotronik leads, revealing the significantly better performance of the latter manufacturer. 